\title{
Extended Jensen's Functional for Diamond Integral via Hermite Polynomial
}

\author{
Rabia Bibi $\mathbb{D},{ }^{1}$ Fazilat Bibi $\mathbb{D}^{2},{ }^{2}$ Ammara Nosheen $\mathbb{D}{ }^{2}$ and Josip Pečarić $\mathbb{D}^{3}$ \\ ${ }^{1}$ Department of Mathematics, Abbottabad University of Science and Technology, Havelian, Abbottabad, Pakistan \\ ${ }^{2}$ Department of Mathematics \& Statistics, University of Lahore, Sargodha Sub-Campus, Pakistan \\ ${ }^{3}$ Rudn University, 6 Miklukho-Maklay St, Moscow 117198, Russia
}

Correspondence should be addressed to Rabia Bibi; emaorr@gmail.com

Received 16 April 2021; Accepted 26 July 2021; Published 12 August 2021

Academic Editor: Andrea Scapellato

Copyright (c) 2021 Rabia Bibi et al. This is an open access article distributed under the Creative Commons Attribution License, which permits unrestricted use, distribution, and reproduction in any medium, provided the original work is properly cited.

In this paper, with the help of Hermite interpolating polynomial, extension of Jensen's functional for $n$-convex function is deduced from Jensen's inequality involving diamond integrals. Special Hermite conditions, including Taylor two-point formula and Lagrange's interpolation, are also deployed to find further extensions of Jensen's functional. The paper also includes discussion on bounds for Grüss-type inequality, Ostrowski-type inequality, and Čebyšev functional associated with newly defined Jensen's functional.

\section{Introduction}

John Jensen proved Jensen's inequality in [1]. It serves as a tool in discrete and continuous analysis for generating classical inequalities. A discrete variant is as below:

$$
\chi\left(\frac{\sum_{m=1}^{n} g_{m} z_{m}}{\sum_{m=1}^{n} g_{m}}\right) \leq \frac{\sum_{m=1}^{n} g_{m} \chi\left(z_{m}\right)}{\sum_{m=1}^{n} g_{m}},
$$

where $\left(z_{1}, \cdots, z_{n}\right) \in S$, $S$ is interval in $\mathbb{R}\left(g_{1}, \cdots, g_{n}\right) \in \mathbb{R}_{+}^{n}$ (i.e., nonnegative weights are taken into account in this inequality), and function $\chi: S \longrightarrow \mathbb{R}$ is a convex on $S$. Steffensen in [2] extended it by using negative weights.

Integral representation of Jensen's inequality in [3] is as follows: If $\tau \in C\left(\left[a_{1}, a_{2}\right],\left(a_{3}, a_{4}\right)\right)$ and $\chi \in C\left(\left(a_{3}, a_{4}\right), \mathbb{R}\right)$ are convex, then

$$
\chi\left(\frac{\int_{a_{1}}^{a_{2}} \tau(\mathfrak{g}) d \mathfrak{I}}{a_{2}-a_{1}}\right) \leq \int_{a_{1}}^{a_{2}} \frac{\chi(\tau(\mathfrak{I})) d \mathfrak{g}}{a_{2}-a_{1}} .
$$

The researchers have devised several new functions for refinements of Jensen's discrete/integral inequalities. For instance, in [4-7], improvements of the operated version of Jensen's inequality are given. In [8], Aras-Gazic et al. generalized Jensen's inequality via the Hermite polynomial.

Several researchers discussed and applied these inequalities on time scales. In [9], Anwar et al. proved Jensen's inequality for delta integrals.

Suppose $a_{1}, a_{2} \in \mathbb{T}$ s.t. $a_{1}<a_{2}$. Let $\partial \in C_{r d}\left(\left[a_{1}, a_{2}\right]_{\mathbb{T}}, \mathbb{R}\right)$ assures $\int_{a_{1}}^{a_{2}}|\partial(\mathfrak{g})| \Delta \mathfrak{g}>0$. If $\chi \in C(S, \mathbb{R})$ is convex, an interval $S \subset \mathbb{R}$ and $\hbar \in C_{r d}\left(\left[a_{1}, a_{2}\right]_{\mathbb{T}}, S\right)$, then

$$
\chi\left(\frac{\int_{a_{1}}^{a_{2}}|\partial(\mathfrak{I})| \hbar(\mathfrak{I}) \Delta \mathfrak{I}}{\int_{a_{1}}^{a_{2}}|\partial(\mathfrak{I})| \Delta \mathfrak{I}}\right) \leq \frac{\int_{a_{1}}^{a_{2}}|\partial(\mathfrak{I})| \chi(\hbar(\mathfrak{I})) \Delta \mathfrak{I}}{\int_{a_{1}}^{a_{2}}|\partial(\mathfrak{I})| \Delta \mathfrak{I}} .
$$

Under a similar hypothesis, in [10], by replacing the delta integral with the nabla integral, the same results are obtained.

Sheng et al. in [11] presented the convex combination of the delta and nabla integrals named as diamond alpha integrals, where $\alpha \in[0,1]$. For $\alpha=1$, we get the usual delta integral and nabla integral for $\alpha=0$. In [12], following Jensen's inequality for the diamond alpha integral is given. 
Suppose a time scale $\mathbb{T} a_{1}, a_{2} \in \mathbb{T}$ s.t. $a_{1}<a_{2}$, and $E \in \mathbb{R}$ is an interval $\hbar \in C_{r d}\left(\left[a_{1}, a_{2}\right]_{\mathbb{T}}, E\right)$, and $\partial \in C\left(\left[a_{1}, a_{2}\right], \mathbb{R}\right)$ so that

$$
\int_{a_{1}}^{a_{2}}|\partial(\mathfrak{s})| \nabla_{\alpha} \mathfrak{S}>0
$$

if $\chi \in C(E, \mathbb{R})$ is convex, then

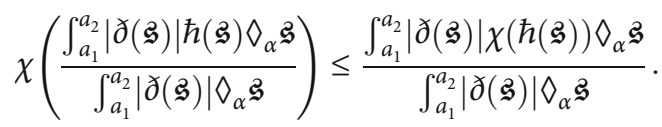

In [13], the authors introduced the more generalized variant of diamond-alpha integrals; termed as the diamond integral, those are of special concern even for $\mathbb{T}=\mathbb{R}$. These integrals get us nearer in building a true symmetric integral on time scales.

In [14], Jensen's inequality for diamond integrals is proved.

Let $a_{1}, a_{2} \in \mathbb{T}$ with $a_{1}<a_{2}, \partial \in C\left(\left[a_{1}, a_{2}\right]_{\mathbb{T}}, \mathbb{R}^{+}\right)$and $\hbar \in C$ $\left(\left[a_{1}, a_{2}\right]_{\mathbb{T}}, S\right)$. Suppose a convex function $\chi \in C(S, \mathbb{R})$ assuring $\int_{a_{1}}^{a_{2}} \partial(u) \Delta u>0$, where $S=\left[m_{1}, m_{2}\right]$ and $m_{1}=\min _{\mathfrak{g} \in\left[a_{1}, a_{2}\right]_{\pi}} \hbar(\mathfrak{g})$, $m_{2}=\max _{\mathfrak{G} \in\left[a_{1}, a_{2}\right]_{\mathbb{T}}} \hbar(\mathfrak{g})$, then

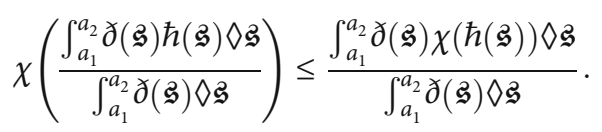

Jensen's-type linear functional defined on $\mathbb{T}$ is given as the following.

Let $a_{1}, a_{2} \in \mathbb{T}$ with $a_{1}<a_{2}$ and $\partial \in C\left(\left[a_{1}, a_{2}\right]_{\mathbb{T}}, \mathbb{R}\right), \hbar \in C$ $\left(\left[a_{1}, a_{2}\right]_{\mathbb{T}}, S\right)$ and $\zeta \in C(S, \mathbb{R})$ satisfying $\int_{a_{1}}^{a_{2}} \partial(\mathfrak{g}) \diamond \mathfrak{g} \neq 0$, where $S=\left[m_{1}, m_{2}\right]$ and $m_{1}=\min _{\mathfrak{g} \in\left[a_{1}, a_{2}\right]_{\mathbb{T}}} \hbar(\mathfrak{g}), m_{2}=\max _{\mathfrak{g} \in\left[a_{1}, a_{2}\right]_{\mathbb{T}}} \hbar(\mathfrak{g})$; then,

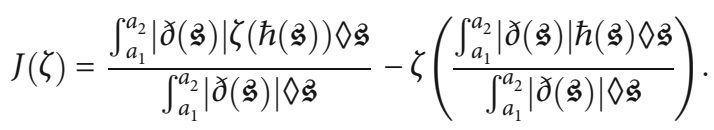

Remark 1 . Inequality (6) implies that $J(\zeta) \geq 0$ for the family of convex mappings and $J(\zeta)=0$ for identity or constant functions.

The present study is aimed at extending (7) for the $n$-convex function with some types of interpolations introduced by Hermite. In the next section, after defining diamond derivative and integral, we recall the Hermite interpolating polynomial along with some of its special forms. Section 3 consists of the paper's main results, and finally, concluding remarks are given in the last section.

\section{Preliminaries}

2.1. Some Essentials Form Diamond Calculus. A time scale T is a nonempty closed subset of $\mathbb{R}$. It may be connected or not; keeping the time scales' disconnection under consideration, forward and backward jump operators $\sigma, \rho: \mathbb{T} \longrightarrow \mathbb{T}$, are defined by $\sigma(\hbar)=\inf \{s \in \mathbb{T}: s>\hbar\}$, and $\rho(\hbar)=\sup \{s \in \mathbb{T}: s$ $<\hbar\}$. In general, $\sigma(\hbar) \geq \mathrm{y}$ and $\rho(\hbar) \leq \hbar$. The mappings $\mu$, $v: \mathbb{T} \longrightarrow[0,+\infty)$ defined by $\mu(\hbar)=\sigma(\hbar)-\hbar$, and $v(\hbar)=\hbar$ $-\rho(\hbar)$ are called in the sequal the forward and backward graininess functions. For the classification of points on time scales, for any $\hbar \varepsilon \mathbb{T}$,

(i) if $\rho(\hbar)=\hbar$, then $\hbar$ is left dense

(ii) if $\sigma(\hbar)=\hbar$, then $\hbar$ is right dense

(iii) if $\rho(\hbar)<\hbar$, then $\hbar$ is left scattered

(iv) if $\sigma(\hbar)>\hbar$, then right scattered

(v) if $\rho(\hbar)=\hbar$ and $\sigma(\hbar)=\hbar$, then $\hbar$ is dense

(vi) if $\rho(\hbar)<\hbar$ and $\sigma(\hbar)>\hbar$, then $\hbar$ is isolated

A mapping $\rho: \mathbb{T} \longrightarrow \mathbb{R}$ is said to be $\mathrm{rd}$-continuous if

(i) it is continuous $\forall \hbar \in \mathbb{T}$ s.t. $\sigma(\hbar)=\hbar$

(ii) left-sided limit is finite $\forall \hbar \in \mathbb{T}$ s.t. $\rho(\hbar)=\hbar$

A set of such functions is denoted by $C_{r d}$.

Definition 2. Let $\Lambda: \mathbb{T} \longrightarrow \mathbb{R}$ be a mapping and $\hbar \in \mathbb{T}_{k}^{k}$ define $\Lambda^{\diamond}(\hbar)$ (presumed it as a finite positive number) having characteristic that, for given $\varepsilon>0$, there exists neighbourhood $W$ of $\hbar$ (i.e. $W=\hbar-s, \hbar+s) \cap T$ ) for some $\delta>0$ such that

$$
\begin{aligned}
& \mid\left[\Lambda^{\sigma}(\hbar)-\Lambda(s)+\Lambda(2 \hbar-s)-\Lambda^{\rho}(\hbar)\right] \\
& \quad-\Lambda^{\diamond}(\hbar)[\sigma(\hbar)+2 \hbar-2 s-\rho(\hbar)]|\leq \varepsilon| \sigma(\hbar)+2 \hbar-2 s-\rho(\hbar) \mid
\end{aligned}
$$

holds. For all $s \in W$ for which $2 \hbar-s \in W$. Then $\Lambda^{\diamond}(r)$ is known as diamond derivative of $\Lambda$ at $\hbar$.

Definition 3. Let $\varrho: \mathbb{T} \longrightarrow \mathbb{R}$ and $a_{1}, a_{2} \in \mathbb{T}$ be a function. The diamond integral of $\mathrm{Q}$ from $a_{1}$ to $a_{2}$ is given by

$$
\int_{a_{1}}^{a_{2}} \mathrm{\varrho}(\hbar) \Delta \hbar:=\int_{a_{1}}^{a_{2}} \gamma(\hbar) \mathrm{\varrho}(\hbar) \Delta \hbar+\int_{a_{1}}^{a_{2}}(1-\gamma(\hbar)) \mathrm{\varrho}(\hbar) \nabla(\hbar),
$$

for all $\hbar \in \mathbb{T}$.

Let $\gamma \varrho$ and $(1-\gamma) \varrho$ be delta and nabla integrable on $[r, s]_{\mathbb{T}}$ , respectively. It is to be noted that the antiderivative is absent for diamond combined derivatives. For $\hbar \in \mathbb{T}_{k}^{k},\left(\int_{b}^{s} \mathrm{Q}(\hbar) \diamond\right)^{\diamond}$ $\neq \mathrm{Q}(s)$, in general. The fundamental theorem of calculus also does not hold for diamond integrals.

2.2. Results on Hermite Interpolating Polynomial. Let $-\infty<$ $\mu<v<\infty$ and $\mu=a_{1}<\cdots<a_{r}=v(r \geq 2)$ be the given $r$ points. For $\mathfrak{\Im} \in C^{n}[\mu, v]$, there is a $(n-1)^{\text {th }}$ degree polynomial $\sqsubseteq_{H}(\mathfrak{t})$, defined as

$$
\sqsubseteq_{H}(\mathfrak{t})=\sum_{v=1}^{r} \sum_{u=0}^{k_{v}} H_{u v}(\mathfrak{t}) \zeta^{(u)}\left(a_{v}\right)
$$


It satisfies the following Hermite condition:

$$
\sqsubseteq_{H}^{(u)}\left(a_{v}\right)=\zeta^{(u)}\left(a_{v}\right), 0 \leq u \leq k_{v}, 1 \leq v \leq r, \sum_{v=1}^{r} k_{v}+r=n .
$$

$H_{u v}$ represents essential polynomials of the Hermite basis which satisfy the relations:

$$
\begin{aligned}
& H_{u v}^{(p)}\left(a_{d}\right)=0, d \neq v, p=0, \cdots, k_{d}, \\
& H_{u v}^{(p)}\left(a_{v}\right)=\delta_{u p}, p=0, \cdots, k_{v}, \text { for } u=0, \cdots, k_{v},
\end{aligned}
$$

with $d, v=1, \cdots, r$ and

$$
\delta_{u p}=\left\{\begin{array}{l}
1 u=p \\
0 u \neq p
\end{array}\right.
$$

$H_{u v}(\mathbf{t})$ is given by

$H_{u v}(\mathfrak{t})=\left.\frac{1}{u !} \frac{\omega(\mathfrak{t})}{\left(\mathbf{t}-a_{v}\right)^{k_{v}+1-u}} \sum_{k=0}^{k_{v}-u} \frac{1}{k !} \frac{d^{k}}{d \mathfrak{t}^{k}}\left(\frac{\left(\mathbf{t}-a_{v}\right)^{k_{v}+1}}{\omega(\mathfrak{t})}\right)\right|_{\mathbf{t}=a_{v}}\left(\mathbf{t}-a_{v}\right)^{k}$

with

$$
\omega(\mathbf{t})=\prod_{v=1}^{r}\left(\mathbf{t}-a_{v}\right)^{k_{v}+1}
$$

Hermite conditions encompass the following specific cases.

2.2.1. Lagrange Conditions. Let $r=n, k_{v}=0$ for all $v$, where 1 $<v<r$. Then, we have Lagrange polynomial $\sqsubseteq_{L}(\mathfrak{t})$, satisfying

$$
\sqsubseteq_{L}\left(a_{v}\right)=\zeta\left(a_{v}\right), 1 \leq v \leq n
$$

2.2.2. Conditions for Type $(\mathfrak{z}, n-z)$. Let $r=2,1 \leq z \leq n-1$, $k_{1}=\mathfrak{z}-1, k_{2}=n-\mathfrak{z}-1$. Then we have $\sqsubseteq_{(\mathfrak{z}, n)}(\mathfrak{t})$ polynomial, satisfying

$$
\begin{aligned}
& \sqsubseteq_{(z, n)}^{(u)}(\mu)=\zeta^{(u)}(\mu), 0 \leq u \leq z-1, \\
& \coprod_{(z, n)}^{(u)}(v)=\zeta^{(u)}(v), 0 \leq u \leq n-z-1 .
\end{aligned}
$$

2.2.3. Conditions for Taylor's Two-Point Formula. For $n=2$ $z, r=2, k_{1}=k_{2}=z-1$, we have Taylor two-point interpolating polynomial $\sqsubseteq_{2 T}(\mathfrak{t})$, satisfying

$$
\sqsubseteq_{2 T}^{(i)}(\mu)=\zeta^{(u)}(\mu), \sqsubseteq_{2 T}^{(u)}(\nu)=\zeta^{(u)}(\nu), 0 \leq u \leq \mathfrak{z}-1 .
$$

The next theorem is useful for our results and is given in [15].
Theorem 4. Suppose we have $-\infty<\mu<v<\infty$ and $\mu=a_{1}<$ $\cdots<a_{r}=v(r \geq 2)$, and $\mathfrak{I} \in C^{n}[\mu, v]$. Then we have

$$
\zeta(\mathfrak{t})=\sqsubseteq_{H}(\mathfrak{t})+R_{H}(\zeta, \mathfrak{t})
$$

where $\sqsubseteq_{H}(\mathbf{t})$ is the Hermite interpolating polynomial as defined in (10) and $R_{H}(\zeta, \mathfrak{t})$ denotes the remainder given by

$$
R_{H}(\zeta, \mathfrak{t})=\int_{\mu}^{v} G_{H, n}(\mathbf{t}, s) \zeta^{(n)}(s) d s
$$

$$
G_{H, n}(\mathbf{t}, s) \text { is }
$$

$$
G_{H, n}(\mathbf{t}, s)= \begin{cases}\sum_{v=1}^{b} \sum_{u=0}^{k_{v}} \frac{\left(a_{v}-h\right)^{n-u-1}}{(n-u-1) !} H_{u v}(\mathbf{t}), & s \leq \mathbf{t}, \\ -\sum_{v=b+1}^{r} \sum_{u=0}^{k_{v}} \frac{\left(a_{v}-h\right)^{n-u-1}}{(n-u-1) !} H_{u v}(\mathbf{t}), & s \geq \mathbf{t},\end{cases}
$$

for all $a_{b} \leq s \leq a_{b+1}, b=0, \cdots, r$ with $a_{0}=\mu$ and $a_{r+1}=v$.

Remark 5. By imposing the Lagrange conditions, Theorem 4 takes the form

$$
\zeta(\mathfrak{t})=\sqsubseteq_{L}(\mathfrak{t})+R_{L}(\zeta, \mathfrak{t})
$$

Here, $\sqsubseteq_{L}(\mathbf{t})$ represents the Lagrange polynomial, which is

$$
\sqsubseteq_{L}(\mathbf{t})=\sum_{v=1}^{n} \prod_{\substack{k=1 \\ k \neq v}}^{n}\left(\frac{\mathfrak{t}-a_{k}}{a_{v}-a_{k}}\right) \zeta\left(a_{v}\right)
$$

and $R_{L}(\zeta, \mathfrak{t})$ is the remainder, defined by

$$
R_{L}(\zeta, \mathfrak{t})=\int_{\mu}^{v} G_{L}(\mathfrak{t}, s) \zeta^{(n)}(s) d s
$$

with

$$
G_{L}(\mathbf{t}, s)=\frac{1}{(n-1) !}\left\{\begin{array}{l}
\sum_{v=1}^{b}\left(a_{v}-s\right)^{n-1} \prod_{\substack{k=1 \\
k \neq v}}^{n}\left(\frac{\mathbf{t}-a_{k}}{a_{v}-a_{k}}\right) s \leq \mathbf{t}, \\
-\sum_{v=1}^{b}\left(a_{v}-s\right)^{n-1} \prod_{\substack{k=1 \\
k \neq v}}^{n}\left(\frac{\mathbf{t}-a_{k}}{a_{v}-a_{k}}\right) s \leq \mathbf{t},
\end{array}\right.
$$

$$
a_{b} \leq s \leq a_{b+1}, b=1, \cdots, n-1 \text { along } a_{1}=\mu \text { and } a_{n}=v \text {. }
$$

Remark 6. Similarly, by imposing $(z, n-z)$ conditions on Theorem 4, one gets

$$
\zeta(\mathfrak{t})=\sqsubseteq_{(\mathfrak{z}, n)}(\mathbf{t})+R_{(\mathfrak{z}, n)}(\zeta, \mathfrak{t}),
$$


where

$$
\sqsubseteq_{(z, n)}(\mathbf{t})=\sum_{u=0}^{z^{-1}} \xi_{u}(\mathbf{t}) \zeta^{(u)}(\mu)+\sum_{u=0}^{n-z^{-1}} \eta_{u}(\mathfrak{t}) \zeta^{(u)}(v)
$$

with

$$
\xi_{u}(\mathbf{t})=\frac{1}{u !}(\mathbf{t}-\mu)^{u}\left(\frac{\mathbf{t}-v}{\mu-v}\right) \sum_{k=0}^{(n-\mathfrak{z})}\left(\begin{array}{c}
\mathfrak{z}-1-u \\
k
\end{array}\right)\left(\frac{\mathfrak{t}-\mu}{v-\mu}\right)^{k},
$$

$$
\eta_{u}(\mathfrak{t})=\frac{1}{u !}(\mathfrak{t}-v)^{u}\left(\frac{\mathfrak{t}-\mu}{v-\mu}\right)^{\mathfrak{z}} \sum_{k=0}^{n-z-1-u}\left(\begin{array}{c}
\mathfrak{z}+k-1 \\
k
\end{array}\right)\left(\frac{\mathfrak{t}-v}{\mu-v}\right)^{k} .
$$

The remainder $R_{(z, n)}(\zeta, \mathrm{t})$ is

$$
R_{(z, n)}(\zeta, \mathfrak{t})=\int_{\mu}^{\nu} G_{(m, n)}(\mathbf{t}, s) \zeta^{(n)}(s) d s
$$

with

$$
G_{(z, n)}(\mathfrak{t}, s)=\left\{\begin{array}{l}
\sum_{v=0}^{z-1}\left[\sum_{l=0}^{z-1-v}\left(\begin{array}{c}
n-\mathfrak{z}+l-1 \\
l
\end{array}\right)\left(\frac{\mathfrak{t}-v}{v-\mu}\right)^{l}\right] \times \frac{(\mathfrak{t}-\mu)^{v}(\mu-s)^{n-v-1}}{v !(n-v-1) !}\left(\frac{v-\mathfrak{t}}{v-\mu}\right)^{n-\mathfrak{z}} \mu \leq s \leq \mathfrak{t} \leq v, \\
-\sum_{u=0}^{n-z-1}\left[\sum_{\mathfrak{r}=0}^{n-z-u-1}\left(\begin{array}{c}
\mathfrak{z}+\mathfrak{r}-1 \\
\mathfrak{r}
\end{array}\right)\left(\frac{v-\mathfrak{t}}{v-\mu}\right)^{\mathfrak{r}}\right] \times \frac{(\mathfrak{t}-v)^{u}(v-s)^{n-u-1}}{u !(n-u-1) !}\left(\frac{\mathfrak{t}-\mu}{v-\mu}\right)^{e} \mu \leq \mathfrak{t} \leq s \leq v .
\end{array}\right.
$$

Remark 7. Theorem 4 in the form of the Taylor two-point formula becomes

$$
\zeta(\mathfrak{t})=\sqsubseteq_{2 T}(\mathfrak{t})+R_{2 T}(\zeta, \mathfrak{t}),
$$

where the Taylor two-point interpolating polynomial $\subseteq_{2 T}(\mathfrak{t})$ is defined by

$$
\begin{aligned}
\sqsubseteq_{2 T}(\mathfrak{t})= & \sum_{u=0} \sum_{k=0}^{z-1}\left(\begin{array}{c}
z-1-u \\
k
\end{array}\right)\left[\frac{(\mathfrak{t}-\mu)^{u}}{u !}\left(\frac{\mathfrak{t}-v}{\mu-v}\right)^{z}\left(\frac{\mathfrak{t}-\mu}{v-\mu}\right)^{k} \zeta^{(u)}(\mu)\right. \\
& \left.+\frac{(\mathfrak{t}-v)^{u}}{u !}\left(\frac{\mathfrak{t}-\mu}{v-\mu}\right)^{z}\left(\frac{\mathfrak{t}-v}{\mu-v}\right)^{k} \zeta^{(u)}(v)\right]
\end{aligned}
$$

and $R_{2 T}(\zeta, \mathfrak{t})$ is

$$
R_{2 T}(\zeta, \mathfrak{t})=\int_{\mu}^{\nu} G_{2 T}(\mathbf{t}, s) \zeta^{(n)}(s) d s,
$$

with

$$
G_{2 T}(\mathbf{t}, s)=\left\{\begin{array}{c}
\frac{(-1)^{\mathfrak{z}}}{(2 \mathfrak{z}-1) !} l^{\mathfrak{z}}(\mathbf{t}, s) \sum_{v=0}^{z-1}\left(\begin{array}{c}
\mathfrak{z}-1+v \\
v
\end{array}\right)(\mathfrak{t}-s)^{\mathfrak{z}-1-v} \mathbf{r}^{v}(\mathbf{t}, s), \quad s \leq \mathbf{t}, \\
\frac{(-1)^{\mathfrak{z}}}{(2 \mathfrak{z}-1) !} \mathfrak{r}^{\mathfrak{z}}(\mathbf{t}, s) \sum_{v=0}^{b^{-1}}\left(\begin{array}{c}
\mathfrak{z}-1+v \\
v
\end{array}\right)(s-\mathfrak{t})^{\mathfrak{z}^{-1-v}} l^{v}(\mathbf{t}, s) \quad \mathbf{t} \leq s,
\end{array}\right.
$$

where $l(\mathbf{t}, s)=(s-\mu)(v-\mathfrak{t}) / v-\mu, \mathfrak{r}(\mathbf{t}, s)=l(\mathbf{t}, s)$, for all $\mathbf{t}, s$ $\in[\mu, v]$.

\section{Main Results}

3.1. Extension of Jensen's Functional via Hermite Polynomial. Here, we prove our key identity regarding the extension of Jensen's functional.

Theorem 8. Let $n \in \mathbb{N}$ and $\zeta \in C^{n}[\mu, v]$ be a convex function. Then for all $\mathrm{t} \in[\mu, v]$, we have

$$
J(\zeta)=\sum_{v=1}^{r} \sum_{u=0}^{k_{v}} J\left(H_{u v}(\mathbf{t})\right) \zeta^{(u)}\left(a_{v}\right)+\int_{\mu}^{v} J\left(G_{H, n}(\mathbf{t}, s)\right) \zeta^{n}(s) d s,
$$

where $J\left(G_{H, n}(t, s)\right)$ is defined as

$J\left(G_{H, n}(\mathbf{t}, s)\right)=\frac{\int_{a_{1}}^{a_{2}}|h(\mathbf{t})| G_{H, n}(f(\mathfrak{t}), s) \diamond \mathbf{t}}{\int_{a_{1}}^{a_{2}}|h(\mathbf{t})| \diamond \mathfrak{t}}-G_{H, n}\left(\frac{\int_{a_{1}}^{a_{2}}|h(\mathfrak{t})| f(\mathfrak{t}) \diamond \mathfrak{t}}{\int_{a_{1}}^{a_{2}}|h(\mathbf{t})| \diamond \mathfrak{t}}, s\right)$.

Proof. Substitute (19) in (7), then the linearity of $J(\cdot)$ gives us (36).

Remark 9. Instead of using (19) in (7), if we use (22), (26), or (32) in (7), then extended results similar to Theorem 8 are obtained for Lagrange conditions, type $(\mathfrak{z}, n-\mathfrak{z})$ conditions, 
and two-point Taylor conditions, respectively, in the following form:

$$
\begin{aligned}
J(\zeta)= & \sum_{v=1}^{n} \zeta\left(a_{v}\right) J\left(\prod_{\substack{k=1 \\
k \neq v}}^{n}\left(\frac{\mathbf{t}-a_{k}}{a_{v}-a_{k}}\right)\right)+\int_{\mu}^{v} J\left(G_{L}(\mathbf{t}, s)\right) \zeta^{n}(s) d s, \\
J(\zeta)= & \sum_{u=0}^{z^{-1}} \zeta^{(u)}(\mu) J\left(\xi_{u}(t)\right)+\sum_{u=0}^{n-z-z} \zeta^{(u)}(v) J\left(\eta_{u}(\mathfrak{t})\right) \\
& +\int_{\mu}^{v} J\left(G_{(z, n)}(\mathbf{t}, s)\right) \zeta^{(n)}(s) d s, \\
J(\zeta)= & \sum_{u=0}^{z-1} \sum_{k=0}^{z^{-1-u}}\left(\begin{array}{c}
\mathfrak{z}+k-1 \\
k
\end{array}\right)\left[J\left(\frac{(\mathbf{t}-\mu)^{u}}{u !}\left(\frac{\mathbf{t}-v}{\mu-v}\right)^{z}\left(\frac{\mathbf{t}-\mu}{v-\mu}\right)^{k}\right)\right. \\
& \left.\cdot \zeta^{(u)}(\mu)+J\left(\frac{(\mathbf{t}-v)^{u}}{u !}\left(\frac{\mathbf{t}-\mu}{v-\mu}\right)^{z}\left(\frac{\mathbf{t}-v}{\mu-v}\right)^{k}\right) \zeta^{(u)}(v)\right] \\
& +\int_{\mu}^{v} J\left(G_{(2 T)}(\mathbf{t}, s)\right) \zeta^{(n)}(s) d s .
\end{aligned}
$$

Theorem 10. Presume $n$ be a natural number and $G_{H, n}$ be a Green function given in (21) satisfying

$$
J\left(G_{H, n}(\mathbf{t}, s)\right) \geq 0,
$$

for all $\mathrm{t} \in[\mu, v]$. Then for every $n$-convex $\zeta:[\mu, v] \longrightarrow \mathbb{R}$,

$$
J(\zeta) \geq J(B)
$$

holds, where

$$
B(\mathfrak{t})=\sum_{v=1}^{r} \sum_{u=0}^{k_{v}} \zeta^{(u)}\left(a_{v}\right) H_{u v}(\mathfrak{t}) .
$$

Proof. As the function $\zeta$ is $n$-convex, $\zeta^{n}(t) \geq 0$ for all $\mathfrak{t} \in[\mu, v]$; hence,

$$
J\left(G_{H, n}(\mathfrak{t}, s)\right) \zeta^{n}(\mathfrak{t}) \geq 0 .
$$

Substituting (42) in (36), we have

$$
J(\zeta)-\sum_{v=1}^{r} \sum_{u=0}^{k_{v}} \zeta^{(u)}\left(a_{v}\right) J\left(H_{u v}(\mathfrak{t})\right) \geq 0 .
$$

Since $J$ is linear, (43) can be written as

$$
J(\zeta)-J(B) \geq 0 .
$$

Hence,

$$
J(\zeta) \geq J(B)
$$

Remark 11. (40) is reversed if (39) is reversed.

Utilization of the Lagrange condition produced the result given below.

Corollary 12. Presume $n$ is a natural number and $G_{L}$ defined as in (25) satisfying

$$
J\left(G_{L}(\mathbf{t}, s)\right) \geq 0,
$$

for all $\mathbf{t} \in[\mu, v]$. Then for every $n$-convex function $\zeta:[\mu, v]$ $\longrightarrow \mathbb{R}$

$$
J(\zeta) \geq J(B)
$$

where

$$
B(\mathfrak{t})=\sum_{v=1}^{n} \zeta\left(a_{v}\right) \prod_{\substack{k=1 \\ k \neq v}}^{n}\left(\frac{\mathfrak{t}-a_{k}}{a_{v}-a_{k}}\right) .
$$
result.

The use of type $(z, n-z)$ condition yields the following

Corollary 13. Let $n$ be a natural number and $G_{(\mathfrak{z}, n)}$ be defined in (31). Suppose $\xi_{u}, \eta_{u}$ are defined as in (28) and (29), respectively, satisfying

$$
J\left(G_{(\mathfrak{z}, n)}(\mathbf{t}, s)\right) \geq 0
$$

for all $\mathfrak{t} \in[\mu, v]$. Then for every $n$-convex function $\zeta:[\mu, v]$ $\longrightarrow \mathbb{R}$, we have

$$
J(\zeta) \geq J(B)
$$

where

$$
B(\mathfrak{t})=\sum_{u=0}^{z^{-1}} \zeta^{(u)}(\mu) \xi_{u}(\mathfrak{t})+\sum_{u=0}^{n-z^{-1}} \zeta^{(u)}(v) \eta_{u}(\mathfrak{t}) .
$$

The application of two-point Taylor conditions gives a result as below.

Corollary 14. Let $n \in \mathbb{N}$ and $G_{2 T}$ be the Green function defined as in (35), satisfying

$$
J\left(G_{2 T}(\mathbf{t}, s)\right) \geq 0,
$$

for all $\mathfrak{t} \in[\mu, v]$. Then for $n$-convex function $\zeta:[\mu, v] \longrightarrow \mathbb{R}$,

$$
J(\zeta) \geq J(B)
$$


where

$$
\begin{aligned}
B(\mathfrak{t})= & \sum_{u=0}^{\mathfrak{z}-1} \sum_{k=0}^{\mathfrak{z}-1-u}\left(\begin{array}{c}
\mathfrak{z}+k-1 \\
k
\end{array}\right)\left[\frac{(\mathrm{t}-\mu)^{u}}{u !}\left(\frac{\mathfrak{t}-v}{\mu-v}\right)^{\mathfrak{z}}\right. \\
& \left.\cdot\left(\frac{\mathfrak{t}-\mu}{v-\mu}\right)^{k} \zeta^{(u)}(\mu)+\frac{(\mathfrak{t}-v)^{u}}{u !}\left(\frac{\mathfrak{t}-\mu}{v-\mu}\right)^{\mathfrak{z}}\left(\frac{\mathfrak{t}-v}{\mu-v}\right)^{k} \zeta^{(u)}(v)\right]
\end{aligned}
$$

Remark 15. Technique to prove Corollaries 12-14 is the same as proof of Theorem 10, where we use $n$ convexity of $\zeta$ and linearity of $J$.

Theorem 16. In addition to presumptions of Theorem 10, consider $h \in C\left(\left[a_{1}, a_{2}\right]_{\mathbb{T}}, \mathbb{R}^{+}\right)$and $B:[\mu, v] \longrightarrow \mathbb{R}$ is convex, then

$$
J(\zeta(\mathbf{t})) \geq 0
$$

for all $\mathrm{t} \in[\mu, v]$.

Proof. As $B(\mathfrak{t})$ is convex for all $\mathfrak{t} \in[\mu, v]$, so Remark 1, inferred $J(B(\mathfrak{t})) \geq 0$. Hence, (40) implies $J(\zeta(\mathfrak{t})) \geq 0$.

Remark 17. Relation (55) in Theorem 16 is an extension of Jensen's inequality (6).

3.2. Bounds for Identities Associated to Generalization of Jensen's Functional. Here, we utilize Čebyšev functional and Grüss-type inequalities to present few important results. Let two functions $g_{1}, g_{2}:[2, j] \longrightarrow \mathbb{R}$ be Lebesgue integrable, and the Čebyšev functional is

$$
\begin{aligned}
\left|Y\left(g_{1}, g_{2}\right)\right|= & \frac{1}{J-2} \int_{2}^{\jmath} g_{1}(\mathbf{t}) g_{2}(\mathbf{t}) d \mathbf{t}-\frac{1}{J-2} \int_{i}^{\jmath} g_{1}(\mathbf{t}) d \mathbf{t} \\
& \cdot \frac{1}{J-2} \int_{2}^{\jmath} g_{2}(\mathbf{t}) d \mathbf{t} .
\end{aligned}
$$

The next two theorems are given in [16].

Theorem 18. Let $g_{1}, g_{2}:[2, j] \longrightarrow \mathbb{R}$ be functions such that $g_{1}$ is Lebesgue integrable and $g_{2}$ be the function which is absolutely continuous along $(\cdot-2)(j-\cdot)\left[g_{2}^{\prime}\right]^{2} \in L[2, j]$. Then, we have

$\left|Y\left(g_{1}, g_{2}\right)\right| \leq \frac{1}{\sqrt{2}}\left[Y\left(g_{1}, g_{1}\right)\right]^{1 / 2} \frac{1}{\sqrt{j-i}}\left(\int_{2}^{\jmath}(y-\imath)(j-y)\left[g_{2}{ }^{\prime}(y)\right]^{2} d y\right)^{1 / 2}$.

$1 / \sqrt{2}$ in (57) is possibly the best constant.

Theorem 19. Let $g_{1}, g_{2}:[2, j] \longrightarrow \mathbb{R}$ be functions, such that $g_{1}$ is absolutely continuous with $g_{1}^{\prime} \in L_{\infty}[2,3]$ and $g_{2}$ is mono- tonic nondecreasing on $[2, J]$, then

$$
\left|Y\left(g_{1}, g_{2}\right)\right| \leq \frac{1}{2(j-i)}\left\|g_{1}^{\prime}\right\|_{\infty} \int_{2}^{l}(y-i)(j-y) d g_{2}(y)
$$

The best possible constant is $1 / 2$ in (58).

We denote

$$
\psi(s)=J\left(G_{H, n}(x, s)\right) .
$$

The Čebyšev functional is given as

$$
Y(\psi, \psi)=\frac{1}{v-\mu} \int_{\mu}^{v} \psi^{2}(s) d s-\left(\frac{1}{v-\mu} \int_{\mu}^{v} \psi(s) d s\right)^{2}
$$

Theorem 20. Let $\zeta:[\mu, v] \longrightarrow \mathbb{R}$ be such that $\zeta \in C^{n}[\mu, \nu]$ for $n \in \mathbb{N}$ with $(\cdot-\mu)(\nu-\cdot)\left[\zeta^{(n+1)}\right]^{2} \in L[\mu, v]$ and $\mathrm{t} \in[\mu, v]$ and $G_{H, n}, \psi$ and $Y$ are defined in (21), (59), and (60), respectively. Then,

$$
\begin{aligned}
J(\zeta)= & \sum_{v=1}^{r} \sum_{u=0}^{k_{v}} \zeta^{(u)}\left(a_{v}\right) J\left(H_{u v}(x)\right)+\frac{\zeta^{(n-1)}(\nu)-\zeta^{(n-1)}(\mu)}{\nu-\mu} \int_{\mu}^{v} \psi(s) d s \\
& +\Re_{n}(\mu, v ; \zeta),
\end{aligned}
$$

where the remainder satisfies $\mathfrak{R}_{n}(\mu, v ; \zeta)$ the estimation

$$
\left|\Re_{n}(\mu, v ; \zeta)\right| \leq[Y(\psi, \psi)] \sqrt[1 / 2]{\frac{\nu-\mu}{2}}\left|\int_{\mu}^{v}(s-\mu)(\nu-s)\left[\zeta^{(n+1)}(s)\right]^{2} d s\right|^{1 / 2} .
$$

Proof. We use Theorem 18 for $g_{1} \longrightarrow \psi$ and $g_{2} \longrightarrow \zeta^{n}$, to obtain

$$
\begin{aligned}
& \left|\frac{1}{v-\mu} \int_{\mu}^{v} \psi(s) \zeta^{n}(s) d s-\frac{1}{v-\mu} \int_{\mu}^{v} \psi(s) d s \cdot \frac{1}{v-\mu} \int_{\mu}^{v} \zeta^{n}(s) d s\right| \\
& \quad \leq \frac{1}{\sqrt{2}}[Y(\psi, \psi)]^{1 / 2} \frac{1}{\sqrt{\nu-\mu}}\left|\int_{\mu}^{v}(s-\mu)(v-s)\left[\zeta^{(n+1)}(s)\right]^{2} d s\right|^{1 / 2} .
\end{aligned}
$$

Therefore, we have

$$
\begin{aligned}
\frac{1}{v-\mu} \int_{\mu}^{\nu} \psi(s) \zeta^{n}(s) d s & =\frac{1}{(\nu-\mu)^{2}}\left(\zeta^{(n-1)}(\nu)-\zeta^{(n-1)}(\mu)\right) \int_{\mu}^{v} \psi(s) d s, \\
\int_{\mu}^{v} \psi(s) \zeta^{n}(s) d s & =\frac{\zeta^{(n-1)}(v)-\zeta^{(n-1)}(\mu)}{\nu-\mu} \int_{\mu}^{\nu} \psi(s) d s+\Re(\mu, v ; \zeta),
\end{aligned}
$$


where the remainder $\mathfrak{R}(\mu, v ; \zeta)$ satisfy the estimation (62). Now, from the identity (36),

$$
\begin{aligned}
J(\zeta)= & \sum_{v=1}^{r} \sum_{u=0}^{k_{v}} \zeta^{(u)}\left(a_{v}\right) J\left(H_{u v}(\mathfrak{t})\right)+\int_{\mu}^{v} \psi(s) \zeta^{(n)}(s) d s, \\
J(\zeta)= & \sum_{v=1}^{r} \sum_{u=0}^{k_{v}} \zeta^{(u)}\left(a_{v}\right) J\left(H_{u v}(\mathfrak{t})\right)+\frac{\zeta^{(n-1)}(v)-\zeta^{(n-1)}(\mu)}{v-\mu} \\
& \cdot \int_{\mu}^{v} \psi(s) d s+\Re(\mu, v ; \zeta) .
\end{aligned}
$$

The Grüss-type inequality given below can be obtained by using Theorem 19.

Theorem 21. Assume $\zeta:[\mu, v] \longrightarrow \mathbb{R}$ such that $\zeta \in C^{(n)}[\mu, v$ ]$(n \in \mathbb{N})$ and $\zeta^{(2 n+1)} \geq 0$ on $[\mu, v]$. Suppose $\psi$ and $Y$ are defined in (59) and (60), respectively. Then, we have (61) the remainder $\mathfrak{R}(\mu, v: \zeta)$ satisfies the bound

$$
\begin{aligned}
|\Re(\mu, v ; \zeta)| \leq & (\nu-\mu)\left\|\psi^{\prime}\right\|_{\infty} \\
& \times\left[\frac{\zeta^{(n-1)}(v)+\zeta^{(n-1)}(\mu)}{v-\mu}-\frac{\zeta^{(n-2)}(v)-\zeta^{(n-2)}(\mu)}{v-\mu}\right] .
\end{aligned}
$$

Proof. Applying Theorem 19 for $g_{1} \longrightarrow \psi$ and $g_{2} \longrightarrow \zeta^{(n)}$, we have

$$
\begin{gathered}
\left|\frac{1}{v-\mu} \int_{\mu}^{\nu} \psi(s) \zeta^{n}(s) d s-\frac{1}{v-\mu} \int_{\mu}^{\nu} \psi(s) d s \cdot \frac{1}{v-\mu} \int_{\mu}^{\nu} \zeta^{n}(s) d s\right| \\
\leq \frac{1}{2(v-\mu)}\left\|\psi^{\prime}\right\|_{\infty} \int_{\mu}^{v}(s-\mu)(v-s) \zeta^{(n+1)}(s) d s .
\end{gathered}
$$

Since

$$
\begin{aligned}
\int_{\mu}^{\nu}(s-\mu)(\nu-s) \zeta^{(n+1)}(s) d s= & \int_{\mu}^{\nu}[2 s-(\mu+v)] \zeta^{(n)}(s) d s, \\
\int_{\mu}^{\nu}(s-\mu)(v-s) \zeta^{(n+1)}(s) d s= & (\nu-\mu)\left[\zeta^{(n-1)}(v)+\zeta^{(n-1)}(\mu)\right] \\
& -2\left[\zeta^{(n-2)}(v)+\zeta^{(n-2)}(\mu)\right] ;
\end{aligned}
$$

using (36) and (67), we get (66).

Theorem 22. Let all the presumptions of Theorem 8 be satisfied. Suppose $(\breve{i}, \breve{j})$ is a couple of numbers, i.e., $1 \leq \breve{i}, \breve{j} \leq \infty, 1 / \breve{i}$
$+1 / \breve{j}=1$. Suppose $\left|\zeta^{(n)}\right|^{i}:[\mu, v] \longrightarrow \mathbb{R}$ is a function which is $R$-integrable for some $n \geq 2$. Then, we have

$$
\left|J(\zeta)-\sum_{v=1}^{r} \sum_{u=0}^{k_{v}} \zeta^{(u)}\left(a_{v}\right) J\left(H_{u v}(x)\right)\right| \leq\left\|\zeta^{(n)}\right\|_{i}\left(\int_{\mu}^{v}\left|J\left(G_{H}(x, s)\right)\right|^{\grave{j}} d s\right)^{1 / j} .
$$

For $\breve{i} \in[1, \infty]$, the constant on the right of (69) is sharp and suitable when $\breve{i}$ is one.

Proof. Assume $\wp(s)=J\left(G_{n}(\mathbf{t}, s)\right)$. Hölder's inequality and identity (36) give us

$$
\begin{aligned}
\left|J(\zeta)-\sum_{v=1}^{r} \sum_{u=0}^{k_{v}} J\left(H_{u v}(\mathbf{t})\right) \zeta^{(u)}\left(a_{v}\right)\right| & =\left|\int_{\mu}^{v} \wp(s) \zeta^{(n)}(s) d s\right| \\
& \leq\left\|\zeta^{(n)}\right\|_{i}\left(\int_{\mu}^{v}|\wp(\mathfrak{t})|^{\dot{j}} d s\right)^{1 / \check{j}} .
\end{aligned}
$$

Taking $\zeta$ for $\breve{i} \in(1, \infty)$, such that, $\zeta^{n}(s)=\operatorname{sgn} \wp(s)$ $|\wp(s)|^{1 / \breve{i}-1}$. In case of $\breve{i}=\infty$, take $\zeta^{(n)}(s)=\operatorname{sgn} \wp(s)$.

We prove that for $\breve{i}=1$,

$$
\int_{\mu}^{\nu} \wp(s) \zeta^{(n)}(s) d s \leq \max _{s \in[\mu, v]}|\wp(s)|\left(\int_{\mu}^{\nu}\left|\zeta^{(n)}(s)\right| d s\right)
$$

is the suitable inequality. Let $|\wp(s)|$ achieve its maximum at $\mathfrak{p} \in[\mu, v]$. We suppose firstly that $\wp(\ddot{d})>0$. We define $\zeta(s)$ for small enough $\delta$, by

$$
\zeta_{\delta}(s):=\left\{\begin{array}{l}
0, \mu \leq s \leq \mathfrak{p}, \\
\frac{1}{\varepsilon n !}(s-\mathfrak{p})^{n}, \mathfrak{p} \leq s \leq \mathfrak{p}+\delta, \\
\frac{1}{n !}(s-\mathfrak{p})^{n-1}, \mathfrak{p}+\delta \leq s \leq v .
\end{array}\right.
$$

Then, for $\delta$ small enough,

$$
\left|\int_{\mu}^{v} \wp(s) \zeta^{(n)}(s) d s\right|=\left|\int_{\mathfrak{p}}^{\mathfrak{p}+\delta} \wp(s) \frac{1}{\delta} d s\right|=\frac{1}{\delta} \int_{\mathfrak{p}}^{\mathfrak{p}+\delta} \wp(s) d s .
$$

Now, from the inequality (71), we have

$$
\frac{1}{\delta} \int_{\mathfrak{p}}^{\mathfrak{p}+\delta} \wp(s) d s \leq \wp(\mathfrak{p}) \int_{\mathfrak{p}}^{\mathfrak{p}+\delta} \frac{1}{\delta} d s=\wp(\mathfrak{p}) .
$$




$$
\begin{aligned}
& \text { For } \wp(\mathfrak{p})<0, \text { we set } \zeta_{\delta}(s) \text { as } \\
& \qquad \zeta_{\delta}(s):=\left\{\begin{array}{l}
\frac{1}{n !}(s-\mathfrak{p}-\delta)^{n-1}, \mu \leq s \leq \mathfrak{p}, \\
-\frac{1}{\varepsilon n !}(s-\mathfrak{p}-\delta)^{n}, \mathfrak{p} \leq s \leq \mathfrak{p}+\epsilon, \\
0, \mathfrak{p}+\delta \leq s \leq v
\end{array}\right.
\end{aligned}
$$

The rest of the proof is the same as above.

\section{Conclusion}

Jensen's functional for diamond integral (7) is generalized for $n$-convex functions using the Hermite polynomial in the present article. Different conditions of Hermite polynomial are utilized to describe respective refinements of the functional. In seek of applications, bounds for identities associated to constructed functional are also discussed. Moreover, by defining the functional as difference of right and left sides of extended inequality (40) (where $B$ is defined in (41)), it is possible to study $n$-exponential convexity, exponential convexity, and applications to Stolarsky-type means as discussed by Aras-Gazic et al., in [17] (Sections 5 and 6). This article extends the results of [8] on time scales.

\section{Data Availability}

No data were used to support this study.

\section{Conflicts of Interest}

The authors declare that they have no conflicts of interest.

\section{References}

[1] J. L. W. V. Jensen, "Sur les fonctions convexes et les inégalités entre les valeurs moyennes," Acta Mathematica, vol. 30, pp. 175-193, 1906.

[2] J. F. Steffensen, "On certain inequalities and methods of approximation," Journal of the Institute of Actuaries, vol. 51, no. 3, pp. 274-297, 1919.

[3] D. S. Mitrinovic, Analytic Inequalities, Springer, Verlag Berlin, Hedelberg, New York, 1970.

[4] L. Horvath, K. A. Khan, and J. Pecaric, "Refinement of Jensenâ $€^{\mathrm{TM}} \mathrm{s}$ inequality for operator convex functions," Advances in inequalities and applications, vol. 2014, 2014.

[5] L. Horvath and J. Pecaric, "A refinement of the discrete Jensen's inequality," Mathematical inequalities and applications, vol. 14, no. 4, pp. 777-791, 2011.

[6] H. R. Moradi, S. Furuichi, and M. Sababheh, "A sharper bound for the Jensen's operator inequality," Journal of Nonlinear Convex Analysis, vol. 22, no. 4, pp. 747-758, 2021.

[7] M. Sababheh, H. R. Moradi, and S. Furuichi, "Integrals refining convex inequalities," Bulletin of the Malaysian Mathematical Sciences Society, vol. 43, no. 3, pp. 2817-2833, 2020.

[8] G. Aras-Gazic, V. Culjak, J. Pecaric, and A. Vukelic, "Generalization of Jensen's inequality by Hermite polynomials and related results," in Conference on inequalities and applications $\hat{a}^{T M}$ 14: Book of Abstracts, Institut of Mathematics University of Debrecen, Debrecen, 2014.
[9] M. Anwar, R. Bibi, M. Bohner, and J. Pečarić, "Integral inequalities on time scales via the theory of isotonic linear functionals," Abstract and Applied Analysis, vol. 2011, Article ID 483595, 16 pages, 2011.

[10] U. M. Özkan, M. Z. Sarikaya, and H. Yildirim, "Extensions of certain integral inequalities on time scales," Applied Mathematics Letters, vol. 21, no. 10, pp. 993-1000, 2008.

[11] Q. Sheng, M. Fadag, J. Henderson, and J. M. Davis, “An exploration of combined dynamic derivatives on time scales and their applications," Nonlinear Analysis: Real World Applications, vol. 7, no. 3, pp. 395-413, 2006.

[12] M. R. Sidi Ammi, R. A. Ferreira, and D. F. Torres, "DiamondJensen's inequality on time scales," Journal of Inequalities and Applications, vol. 2008, Article ID 576876, 13 pages, 2008.

[13] A. M. C. Brito da Cruz, N. Martins, and D. F. Torres, "The diamond integral on time scales," Bulletin of the Malaysian Mathematical Sciences Society, vol. 38, no. 4, pp. 1453-1462, 2015.

[14] R. Bibi, A. Nosheen, and J. Pečarić, "Generalization of Jensentype linear functional on time scales via lidstone polynomial," Cogent Mathematics, vol. 4, no. 1, p. 1330670, 2017.

[15] T. Kilgore, R. P. Agarwal, and P. J. Y. Wong, "Error inequalities in polynomial interpolation and their applications," Journal of Approximation Theory, vol. 86, no. 3, pp. 358-359, 1996.

[16] P. Cerone and S. S. Dragomir, "Some new Ostrowski-type bounds for the Čebyšev functional and applications," Journal of Mathematical Inequalities, vol. 8, no. 1, pp. 159-170, 2007.

[17] G. Aras-Gazic, V. Culjak, J. Pecaric, and A. Vukelic, "Generalization of Jensen's inequality by Hermite polynomial and related results," Mathematical Reports, vol. 17, no. 2, pp. 201-223, 2015. 